\title{
Levofloxacin in the treatment of complicated urinary tract infections and acute pyelonephritis
}

\author{
Jessina C McGregor \\ George P Allen \\ David T Bearden \\ Oregon State University College \\ of Pharmacy, Portland, OR, USA
}

Correspondence: Jessina C McGregor Oregon State University/Oregon Health \& Science University College of Pharmacy, Portland Campus at OHSU, 3303 SW Bond Ave., Mail Code: $\mathrm{CHI}$ 2C, Portland, OR 97239, USA

Tel +l 5034944722

Fax +I 5034948797

Email mcgregoj@ohsu.edu

\begin{abstract}
Levofloxacin is a widely used fluoroquinolone approved for the treatment of complicated urinary tract infections and acute pyelonephritis. A comprehensive review of the medical literature identified five publications evaluating levofloxacin for the treatment of either complicated urinary tract infections or acute pyelonephritis. All trials, although variable in their inclusion criteria and levofloxacin dosing strategies, reported microbiologic, clinical, and safety-related outcomes. High microbiologic eradication rates, ranging from $79.8 \%$ to $95.3 \%$, were observed in all studies. Escherichia coli was the most commonly isolated uropathogen. Data on levofloxacin resistance, both at baseline and after therapy, were limited. Clinical success was observed to range from $82.6 \%$ to $93 \%$ when measured after the completion of therapy. These clinical and microbiologic results were comparable to the fluoroquinolone comparators in all trials. Insufficient data are available to evaluate the outcomes in any meaningful patient subgroups, including catheterized patients, and those with other specific complicating factors. Levofloxacin was well tolerated in these studies, with headache, gastrointenstinal effects, and dizziness being the most commonly reported adverse events. The published data support the use of levofloxacin in complicated urinary tract infections and acute pyelonephritis. Further trials are necessary to evaluate levofloxacin within specific patient sub-populations.
\end{abstract}

Keywords: urinary tract infection, pyelonephritis, levofloxacin

\section{Introduction}

Urinary tract infections are one of the most frequently occurring bacterial infections. In the United States alone, they account for over 7 million office visits and approximately $15 \%$ of community-prescribed antibiotics (Mazzulli 2001; Foxman 2002). While the majority of these infections are uncomplicated, patients with complicated urinary tract infections (cUTI) are, by definition, at greater risk for adverse outcomes. Levofloxacin, a widely-used fluoroquinolone, was approved in the US for the treatment of cUTI and acute pyelonephritis (AP) in 1996 and, more recently, in 2008 a higher dose, short-course regimen was also approved for this indication. This review will examine the clinical data available to evaluate the efficacy and safety of this antimicrobial for cUTI and AP.

\section{Complicated urinary tract infections and pyelonephritis}

Generally, a cUTI is considered to be an infection that occurs in the setting of any factor that predisposes to treatment failure or recurrence (McCue 1999). However, no true consensus has been reached within the medical community as to what specifically defines a cUTI. Traditionally, urinary tract infections in patient populations such as those with diabetes mellitus, pregnant females, and males have been considered to be complicated infections (Anderson 1996). Other descriptions of a cUTI have also included patients who are elderly, have experienced recent instrumentation or antimicrobial treatment, or possess functional or anatomic abnormalities of the genitourinary tract (Hooton and Stamm 1991). Ronald et al proposed an alternate classification scheme, 
in which a cUTI is characterized by the presence of structural abnormalities (urinary obstructions, neurogenic bladder, etc.), metabolic and/or hormonal abnormalities (diabetes mellitus, pregnancy, renal impairment, etc.), impaired host responses (transplant recipients, neutropenic patients, etc.), or infection with an unusual pathogen (including yeasts, fungi, and antimicrobial-resistant bacteria) (Ronald and Harding 1997).

Mandell's Principles and Practice of Infectious Diseases defines cUTIs as urinary tract infections in men, pregnant women, children, hospitalized patients, and patients with functional or structural abnormalities of the urinary tract (Sobel and Kaye 2005).

AP is commonly described as an infection of the upper urinary tract that encompasses fever and flank pain and/or tenderness that are accompanied by dysuria and urinary urgency and frequency, although it is more accurately diagnosed based on the presence of these symptoms along with bacteriuria and acute renal infection (Sobel and Kaye 2005). AP was formerly treated largely on an inpatient basis, but a recent trend towards outpatient management of this condition has been noted (Czaja et al 2007). As seen in cUTI, the most common pathogen causing AP is Escherichia coli (Nicolle 1997; Czaja et al 2007).

\section{The role of fluoroquinolones in the management of genitourinary infections}

Currently available fluoroquinolones that are approved by the US Food and Drug Administration (FDA) for the treatment of cUTI and/or AP include ciprofloxacin, levofloxacin, norfloxacin, and ofloxacin. The fluoroquinolones gemifloxacin and moxifloxacin are not approved for either condition. Gatifloxacin was awarded FDA approval for the management of cUTI and AP, but this agent was withdrawn from the US market in 2006. Levofloxacin, approved by the US FDA in 1996, is the $S$ enantiomer of the racemate ofloxacin. The $S$ enantiomer of ofloxacin displays antibacterial activity that is approximately 2 orders of magnitude greater than that of the $R$ enantiomer; this enhanced activity is due to greater binding to and saturation of the binding site on the DNA gyrase enzyme (Morrissey et al 1996). Studies of ofloxacin were not included in the present analysis.

The Infectious Diseases Society of America (IDSA) has published guidelines for the management of acute uncomplicated cystitis and AP (Warren et al 1999). For cases of AP that are manageable on an outpatient basis using oral antimicrobial therapy, the IDSA recommends a fluoroquinolone as the preferred empiric therapy, with trimethoprim/sulfamethoxazole (TMP/SMX) offered as an alternative. In patients that are to be hospitalized, the guidelines recommend the use of intravenous therapy with a fluoroquinolone, an aminoglycoside with or without ampicillin, or an extended-spectrum penicillin with or without an aminoglycoside. After such patients are stabilized, the IDSA recommends that the course of treatment be completed with an oral fluoroquinolone or oral TMP/SMX. Alternatives are recommended for each of the above patient populations if the infecting organism is known to be a Grampositive coccus. Recommended first-line agents for the treatment of uncomplicated cystitis include a fluoroquinolone or TMP/SMX, depending on the local rate of TMP/SMX resistance in E. coli. IDSA is currently working towards assembling guidelines for the treatment of cUTI, and publication is anticipated in late 2008. The European Association of Urology (EAU) has published guidelines that include recommendations for cUTI, which advise the use of fluoroquinolones with mainly renal excretion when empiric therapy is necessary. The EAU recommends avoiding TMP/SMX as first-line treatment because of the increasingly high prevalence of resistance to that antimicrobial agent (Grabe et al 2008).

\section{Fluoroquinolone resistance}

Fluoroquinolones have been extensively used in the management of genitourinary infections, especially acute uncomplicated cystitis. While fluoroquinolone resistance in uropathogens was once rare, resistance in $E$. coli has emerged and continues to increase. Fluoroquinolone resistance occurs through multiple mechanisms including chromosomal point mutations in the genes encoding DNA gyrase and/or topoisomerase iv, mutations that cause decreased expression of outer membrane proteins (OMPs), alterations in the lipopolysaccharide (LPS) component of the cell envelope, and enhanced fluoroquinolone efflux by efflux pumps such as AcrAB (Chenia et al 2006; Chang et al 2007). Plasmid-borne resistance has also recently been discovered, and is caused by protection of DNA gyrase and topoisomerase IV by Qnr-like proteins, including QnrA (Chenia et al 2006). Surveillance of urinary isolates collected between 1989 and 1997 found that fluoroquinolone resistance in $E$. coli was essentially nonexistent during this time frame (Gupta et al 1999a; Gupta et al 1999b). More recently, results of the North American Urinary Tract Infection Collaborative Alliance (NAUTICA) study, a multicenter surveillance study performed between 2003 and 2004 in the US and Canada, reported that overall resistance rates to ciprofloxacin and levofloxacin were $5.5 \%$ and $5.1 \%$, respectively (Zhanel et al 2006). Similar increases 
in fluoroquinolone resistance have been noted in the setting of AP (Czaja et al 2007). Also, among the emerging extendedspectrum beta-lactamase (ESBL)-producing E. coli, higher rates of fluoroquinolone resistance have been reported. In China, levofloxacin resistance among ESBL-producing E. coli was reported in $86 \%$ of isolates (Xiong et al 2002). In the US, one healthcare system reported that $56 \%$ of ESBL-producing E. coli and Klebsiella pneumoniae were resistant to fluoroquinolones (Lautenbach et al 2001).

\section{Levofloxacin pharmacology}

Levofloxacin possesses activity against a variety of uropathogens, including E. coli (Davis and Bryson 1994). The pharmacokinetics of levofloxacin have been reviewed elsewhere, and a comprehensive evaluation of levofloxacin's pharmacokinetics is beyond the scope of this review (Fish and Chow 1997). The bioavailability of levofloxacin is $100 \%$, although absorption is delayed in the presence of food; the degree of protein binding ranges from 24 to $38 \%$. Levofloxacin displays linear pharmacokinetics, and an elimination half-life of 7.6 hours after multiple doses of $500 \mathrm{mg}$. The primary route of elimination is renal, with approximately $71 \%$ of a dose eliminated as unchanged drug within 24 hours. Tissue distribution is extensive; urinary levofloxacin concentrations have been reported to be as high as $128-343 \mathrm{mg} / \mathrm{L}$ (the corresponding plasma maximum concentration $\left(\mathrm{C}_{\max }\right)$ after a single $500 \mathrm{mg}$ dose ranges from 4.5 to $5.2 \mathrm{mg} / \mathrm{L}$ ) (Langtry and Lamb 1998). Levofloxacin also attains adequate penetration into the prostate, with a mean 2.96:1 prostate: plasma concentration ratio, and this agent has demonstrated success in the management of chronic bacterial prostatitis, including equivalent efficacy to ciprofloxacin (Drusano et al 2000; Bundrick et al 2003; Naber et al 2008). The urinary excretion of levofloxacin (84\%) is higher than that of ciprofloxacin (43\%), gatifloxacin (80\%), gemifloxacin (28\%), and moxifloxacin (20\%) (Naber 2001). Renal clearance of levofloxacin (but not the plasma $\mathrm{C}_{\max }$ ) is reduced in the presence of renal impairment.

Few current guidelines describing the optimal treatment of cUTI and AP are available, and the role of levofloxacin in comparison to alternate antimicrobials (including other fluoroquinolones) in the management of these conditions must be further defined. The objective of this review is to provide a summary of the clinical efficacy and safety of levofloxacin in the treatment of cUTI and AP.

\section{Methods}

The PubMed search engine was used to query the National Library of Medicine database using the following search terms: "fluoroquinolone," "levofloxacin," “complicated urinary tract infection," "complicated UTI," "cystitis," "pyelonephritis," "urinary tract infection," and "UTI." This query was also limited to English-language publications. The search criteria were chosen to maximize the sensitivity of the search. The same search criteria were used to query the Cochrane Database of Clinical Trials and the Clinical Trials.gov database. We included all English-language publications of either randomized controlled trials or analytical observational studies of the efficacy of levofloxacin in treating cUTI and/or AP that were published before February 1, 2008. We excluded studies performed in pediatric patients or that included no comparator group. For each publication, the authors' definition of cUTI and AP were used. The citations of all included publications were also considered for inclusion in this review. All publications were assessed for potential duplication of reported data.

For each of the included publications, two authors extracted data on the study design and methods, the definitions of key variables (cUTI, AP, microbiologic eradication, and clinical cure), timing of outcome assessments, efficacy and safety results, infecting pathogens, and the presence and/or development of antibiotic resistance. Data extraction was conducted in this manner so as to evaluate not only the safety and efficacy but also the generalizability of the studies to general practice.

\section{Results}

\section{Study selection}

The PubMed query yielded 385 publications. Of these, five publications met the inclusion and exclusion criteria. However, one of these publications reported the findings of a subset of patients (those with AP) and these data were also subsequently published with the results of the full trial (Klausner et al 2007; Peterson et al 2008). Thus, only the latter publication was included in this review (Peterson et al 2008). Of the publications that were excluded, $86 \%$ were excluded because they were not human subjects-based research (including, for example, systematic reviews), did not include levofloxacin as a study drug, or were not evaluating therapies for cUTI or AP.

The query of the Cochrane Database of Clinical Trials yielded 55 publications. Of these, eight studies were not included because levofloxacin was not evaluated, seven were excluded because they were not studies of cUTI or AP, 13 were non-English publications, and the remaining 27 studies had already been identified for consideration in the PubMed query. Similarly, the ClinicalTrials.gov query 
yielded 60 registered trials (some on-going). Of these trials, 47 were not studies of cUTI or AP, four were conducted in pediatric patients, five were studies not including levofloxacin, and one was an uncontrolled study. Only three trials were identified as having met inclusion and exclusion criteria. The findings of these trials were published, either in whole or in part, in the publications identified through PubMed. Thus, the summary data available through ClinicalTrials.gov were not included in this review. Review of the cited publications yielded one additional, previously unidentified publication that met inclusion and exclusion criteria.

Five publications met all inclusion and exclusion criteria and are included in this review (Klimberg et al 1998; Richard et al 1998a, b; Peng 1999; Peterson et al 2008). Table 1 summarizes the design of the trials reported in these publications. All five publications reported the findings of randomized controlled trials. One reported the results of two such trials (Richard et al 1998b); thus six trials were described among the five publications. We should also note that three of the publications included in this review arise from two trials. More specifically, data from two trials for the subset of AP patients were published in one publication (Richard et al 1998b) and the data pertaining to the cUTI patients from each of these trials were published separately (Klimberg et al 1998; Richard et al 1998a). From this point forward, we will consider these three publications as presenting the results of four distinct trials.

\section{Overview of study designs}

Three trials were conducted in the US (Klimberg et al 1998; Richard et al 1998b; Peterson et al 2008), two trials in the US and Canada (Richard et al 1998b), and one trial was conducted in Taiwan (Peng 1999). The latter was also the only single-center trial. Only two publications specifically stated that the targeted sample size was selected to show equivalence within a margin of $15 \%$ (Richard et al 1998a; Peterson et al 2008). One of these publications did not achieve the sample size targeted by their power calculation (Richard et al 1998a). The remaining trials, which were all of smaller sample sizes, did not specifically describe the statistical power, sample size, or margin of equivalence that the trial was designed to target.

The definition of cUTI varied across the four trials including these patients. The trials performed by Peng and Peterson et al stated only that a diagnosis of cUTI was required, although females in the latter trial were also required to have at least one of the following complicating factors: neurogenic bladder or urinary retention; partial obstruction, renal tumor or fibrosis, distorted urethral structure; and/or intermittent catheterization (Peng 1999; Peterson et al 2008). The studies of cUTI published by Klimberg et al and Richard et al required that all subjects possess an anatomic or functional abnormality of the urinary tract and at least one of the following symptoms: urgency frequency, dysuria, hematuria, or fever or history of fever (Klimberg et al 1998; Richard et al 1998a). Klimberg et al also required that patients present with a baseline urinary sample with more than five urinary white blood cells/high-power field (Klimberg et al 1998). Three trials also required that study participants present with $10^{5}$ or more colony-forming units (cfu)/mL of at least one species of uropathogen (Klimberg et al 1998; Richard et al 1998a; Peterson et al 2008), although Peterson et al excluded patients with more than two species of uropathogen present at baseline (Peterson et al 2008).

Four trials included AP patients. In three of the trials, the definitions of AP were similar and required that patients present with $10^{5}$ or more $\mathrm{cfu} / \mathrm{mL}$ of at least one species of uropathogen and two or more of the following symptoms: fever, flank pain or costovertebral angle tenderness, peripheral white blood cell count (WBC) more than $12,500 / \mathrm{mm}^{3}$ or $10 \%$ or greater bands, and/or WBC casts in the urine, or, in the case of Peterson et al a positive antibody-coated bacteria test (Richard et al 2002; Peterson et al 2008). Similar to the definition of cUTI, Peterson et al required that subjects with AP have at least one of the following symptoms: urgency frequency, dysuria, hematuria, or fever or history of fever (Peterson et al 2008). In contrast, Peng required only a diagnosis of AP (Peng 1999). All trials assessed the outcomes of microbiologic eradication and clinical success. These definitions were similar across all trials (Table 1).

In each of the six trials, another fluoroquinolone was selected as the comparator treatment (ciprofloxacin in three trials, lomefloxacin in two trials, and ofloxacin in one trial). One trial allowed patients to be administered either oral or intravenous formulations. The efficacy and safety results of this trial were not stratified by formulation, although $97 \%$ of the intention to treat group were started on an oral formulation of either levofloxacin or the comparator (Peterson et al 2008). The five remaining trials evaluated oral administration of levofloxacin. Of these, four trials were specifically limited to outpatients and/or patients deemed appropriate candidates for oral therapy (Klimberg et al 1998; Richard et al 1998a, b). Five trials specifically included in their definitions of cUTI and AP that patients' urine cultures at the time of enrollment must contain at least $10^{5}$ $\mathrm{cfu} / \mathrm{mL}$ of a uropathogen (Klimberg et al 1998; Richard et al 


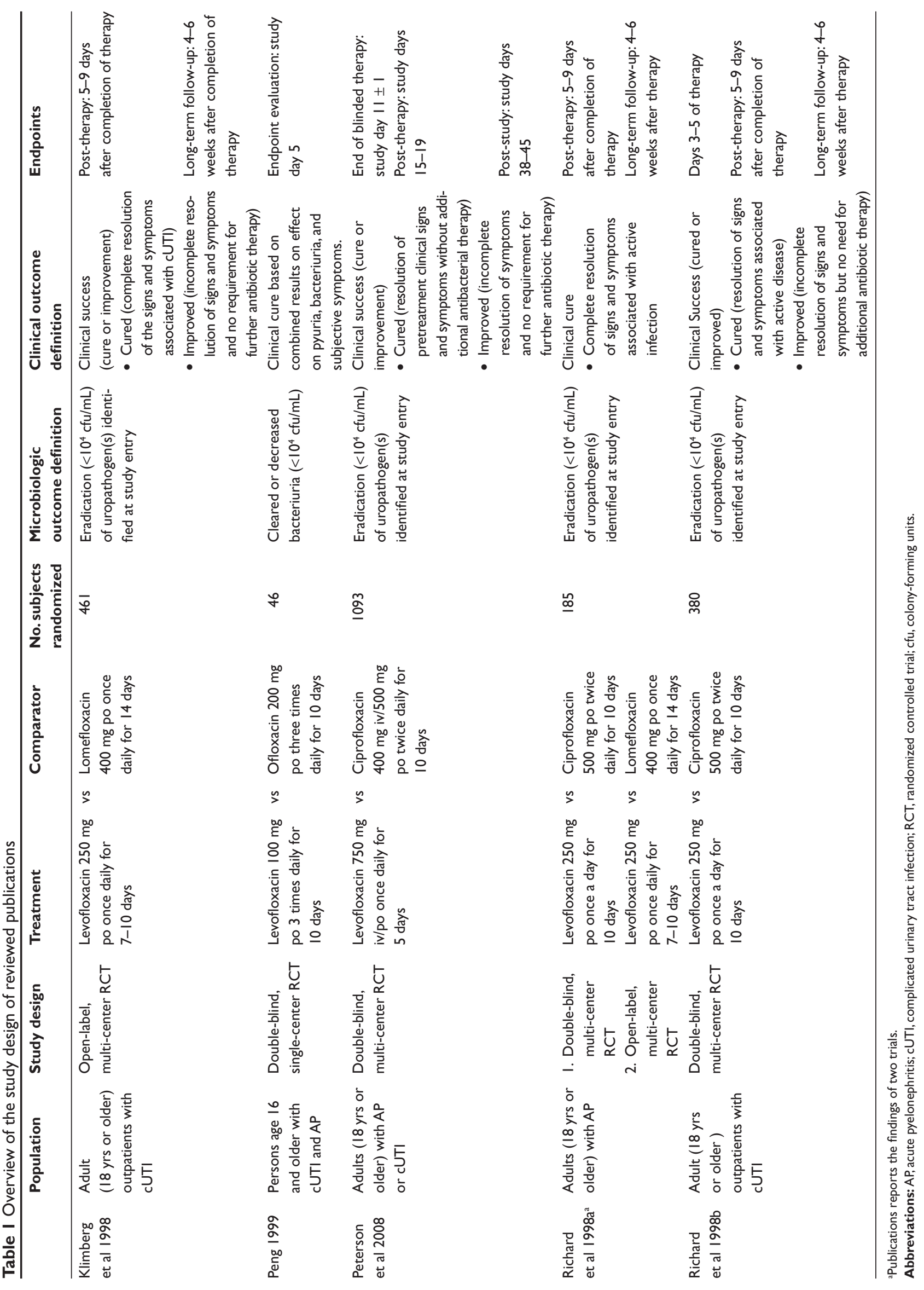


1998a, b; Peterson et al 2008). Four of these trials excluded subjects with previous infections with organisms known to be resistant to any study medication (Klimberg et al 1998; Richard et al 1998a, b). The fifth excluded subjects if the UTI was caused by a pathogen resistant to either study medication (Peterson et al 2008).

Four trials also excluded patients with decreased renal function. Peng excluded patients with renal failure (and other extremely severe underlying diseases) (Peng 1999). Of the two trials described in Richard et al, one excluded all patients with a creatinine clearance less than $50 \mathrm{~mL} / \mathrm{min}$ and the second excluded those with a creatinine clearance less than $20 \mathrm{~mL} / \mathrm{min}$ (Richard et al 1998b). In the latter trial, dosing was altered for those with a creatinine clearance between 20 and $50 \mathrm{~mL} / \mathrm{min}$. This same exclusion criteria (creatinine clearance of less than $20 \mathrm{~mL} / \mathrm{min}$ ) and altered dosing scheme was also applied in the study by Klimberg et al (Klimberg et al 1998). In both publications, no data were provided as to the number of patients receiving altered dosing, nor were the efficacy and safety results reported within this subset of subjects. Richard et al's cUTI trial also excluded patients with creatinine clearance less than $50 \mathrm{~mL} / \mathrm{min}$ (Richard et al 1998a).

\section{Microbiologic response}

Microbiologic eradication rates for levofloxacin were high in all trials. When assessed after the completion of therapy, eradication rates ranging from $79.8 \%$ to $95.3 \%$ were observed (Table 2). These rates were not statistically significantly different from that of the comparator treatments. Peng evaluated microbiologic efficacy only on day 5 of a 10-day course of levofloxacin and observed an eradication rate of $90 \%$, which was also not significantly different from that of the comparator (Peng 1999). Richard et al reported relapse rates from the two trials described in that publication; at 4-6 weeks post-therapy, microbiologic relapse was observed in $13 \%$ of levofloxacin-treated subjects and in $6.5 \%$ of comparator-treated (ciprofloxacin or lomefloxacin) study subjects. The difference was not statistically significant (Richard et al 1998b). In their cUTI trial, Richard et al (1998a) reported a relapse rates of $9 \%$ in the levofloxacintreated group at 4-6 weeks post-therapy, though 38\% of the relapse cases were asymptomatic.

All publications provided information regarding the uropathogens that were most frequently isolated, and in all $E$. coli was the most commonly isolated (average $=61 \%$ ). Other commonly isolated pathogens included $K$. pneumoniae, Pseudomonas aeruginosa, Proteus mirabilis, Enterobacter spp., and Enterococcus spp. While not all studies reported the frequency with which these organisms were isolated, among those that did, $K$. pneumoniae was isolated on average in $16 \%$ of subjects, while the remaining uropathogens were all isolated in $10 \%$ or less of subjects. Among the studies that reported species-specific microbiologic eradication rates, eradication failed on average in $7 \%$ of cases caused by E. coli within levofloxacin-treated subjects (Klimberg et al 1998; Richard et al 1998a; Peterson et al 2008). Peterson et al reported that levofloxacin non-susceptible bacteria were isolated from $6 \%$ of levofloxacin-treated subjects, and that no acquired resistance was observed (Peterson et al 2008). In their cUTI trial, Richard et al (1998a) reported that $9.8 \%$ of isolates tested were not susceptible to levofloxacin (Richard et al 1998a). These were the only publications to provide these data.

As may be expected, the usage of urinary catheters was observed in a number of patients in the included trials. All of the included studies allowed the inclusion of catheterized patients, but only 2 of the studies reported the total number of these patients, which constituted approximately $5 \%$ of the subjects enrolled in these trials (Klimberg et al 1998; Peterson et al 2008). Only one of the trials reported outcomes based on catheter usage. Peterson et al reported a significantly worse microbiologic eradication in the 68 catheterized patients compared to those without catheters (74.1\% vs $89.0 \%$; 95\% CI: $-26.6 \%$ to $-3.3 \%$ ).

\section{Clinical response}

Clinical success was observed to range from $82.6 \%$ to $93 \%$ when measured after the completion of therapy (Table 2). Again, Peng (1999) measured clinical efficacy mid-treatment and observed a clinical success rate of $90 \%$. Statistical testing of the differences in clinical efficacy was only reported in three publications, and none of these reported any significant difference between the levofloxacin and comparator groups (Richard et al 1998a; Peng 1999; Peterson et al 2008). In the remaining two publications, reported clinical efficacy rates were higher for levofloxacin than for the comparator (Klimberg et al 1998; Richard et al 1998b).

\section{Adverse events}

Only Peterson et al (2008) reported the occurrence of serious adverse events (17 events in levofloxacin-treated study subjects). While not all events were detailed in the publication, one allergic reaction and one death were reported. The death was not found to be treatment-related. Adverse event rates were similar for levofloxacin and comparators in all 
Table 2 Summary of reported microbiologic and clinical efficacy data

\begin{tabular}{|c|c|c|c|c|c|c|}
\hline & \multirow[t]{2}{*}{ Condition } & \multicolumn{2}{|c|}{$\begin{array}{l}\text { Efficacy after completion } \\
\text { of therapy }\end{array}$} & \multirow[t]{2}{*}{$\begin{array}{l}\text { No. subjects } \\
\text { evaluated }\end{array}$} & \multirow[t]{2}{*}{ Days post-therapy } & \multirow{2}{*}{$\begin{array}{l}\text { Significantly } \\
\text { different from } \\
\text { comparator(s)? }\end{array}$} \\
\hline & & Microbiologic & Clinical & & & \\
\hline Klimberg et al 1998c & cUTI & $95.3 \%$ & $93.0 \%$ & 171 & $5-9$ & $\mathrm{No}^{f}$ \\
\hline Peng 1999d & $\mathrm{AP}$ and $\mathrm{cUTI}$ & $90.0 \%$ & $90.0 \%$ & 20 & -5 & No \\
\hline Peterson et al $2008^{\mathrm{b}}$ & AP and cUTI & $79.8 \%$ & $82.6 \%$ & 317 & $5-7$ & No \\
\hline Richard et al I998a $a^{\mathrm{c}, \mathrm{e}}$ & AP & $94.0 \%$ & $92.0 \%$ & 89 & $5-9$ & Nog \\
\hline Richard et al I998b & cUTI & $91.0 \%$ & $92.0 \%$ & 126 & $5-9$ & No \\
\hline
\end{tabular}

alpha $=0.05$.

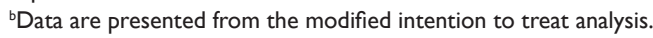

'Data are presented from the microbiologically evaluable study subjects; efficacy not reported for intention to treat or modified intention to treat groups.

No end of therapy endpoint assessed. Data presented was collected on day 5 of therapy.

ePublication reports the findings of two trials; average efficacy presented here.

'Statistical comparison performed only for microbiologic efficacy outcome.

gNo statistical comparisons reported; however, microbiologic and clinical efficacy rates for levofloxacin were higher than for the comparators.

Abbreviations: AP, acute pyelonephritis; CUTI, complicated urinary tract infection.

publications. Only one publication specifically reported that no statistically significant differences in individual adverse event rates were found between treatment groups (Peterson et al 2008). The most commonly observed adverse events across all of the trials include: headache, diarrhea, dyspepsia, nausea, dyspnea, vaginitis, flatulence, and dizziness. Laboratory test abnormalities were infrequently reported. Klimberg et al (1998) reported that changes in clinical laboratory results occurred infrequently and were comparable across groups. Peng (1999) described patients receiving levofloxacin who experienced elevated alanine aminotransferase, potassium, blood urea nitrogen, and hemoglobin, but these laboratory changes were not attributed to levofloxacin therapy. No other studies described laboratory value alterations.

\section{Acute pyelonephritis versus complicated UTI}

Four of the trials included in this review included patients with cUTI. However, two of these trials included patients with $\mathrm{AP}$, and did not provide results stratified by infection. Of these two studies, that of Peng (1999) consisted primarily of AP patients (16/20 in the levofloxacin arm and 19/26 in the ofloxacin arm), while Peterson et al (2008) enrolled primarily cUTI patients (391/537 in the levofloxacin arm and 391/556 in the ciprofloxacin arm). The average levofloxacin efficacy rates for the two publications reporting efficacy for cUTI alone was $93.5 \%$ for microbiologic eradication and $92.6 \%$ for clinical success (Klimberg et al 1998; Richard et al 1998a). While Peterson et al did not report stratified efficacy results, a second publication by the authors (that was not included in this review due to duplicity) reports the results for AP patients alone. This publication also found levofloxacin to be equivalent to the comparator (ciprofloxacin) in both safety and efficacy (Klausner et al 2007). The average levofloxacin efficacy rates for this publication and Richard et al's report of two AP trials were $88.3 \%$ for microbiologic eradication and $88.5 \%$ for clinical success. Also noteworthy is that Peterson et al (2008) is the only trial of high-dose, short duration levofloxacin therapy. This trial observed efficacy rates that were $12.8 \%$ lower on average for microbiologic eradication and $9.2 \%$ lower on average for clinical success than reported by the other publications. As described above, this was also the only trial to report the occurrence of serious adverse events among levofloxacin-treated study subjects.

\section{Discussion}

The objective of this review was to provide a summary of the existing efficacy and safety data pertaining to the use of levofloxacin for the treatment of cUTI and AP. While our search of the literature revealed only five publications meeting the criteria for inclusion, all reported generally high microbiologic and clinical efficacy rates with minimal occurrences of serious adverse events. Despite this, the paucity of data in this area leaves several questions as to the generalizability of these trials.

The US FDA approves medications for the indication of urinary tract infections, including pyelonephritis, under two categories: (1) uncomplicated urinary tract infections and (2) complicated urinary tract infections and pyelonephritis. The trials included in this review used definitions generally consistent with this classification. In contrast to this, the IDSA has utilized a relatively more complex categorization scheme that recommends the use of the following categories in the evaluation of anti-infectives for urinary tract 
infections: (1) acute uncomplicated UTI in women, (2) acute uncomplicated pyelonephritis, (3) complicated UTI and UTI in men, (4) asymptomatic bacteriuria, and (5) recurrent UTI (antimicrobial prophylaxis) (Rubin et al 1992). While the FDA acknowledges that AP can be either an uncomplicated or complicated disease, it justifies the aggregation of all AP with cUTI by noting that antibiotic dosing regimens for these conditions are generally similar (US Department of Health and Human Services et al 1998). Nevertheless, clinical and/or microbiologic responses may vary between patients with different complicating factors, including such factors as physical obstruction of the urinary tract, male gender, and immunosuppression. These differences may stem from the need for additional interventional therapies to ensure the resolution of the infection. For example, cUTI patients with anatomical abnormalities may require surgery, and for those with neurogenic bladders, catheterization may be necessary to manage urination (Matsumoto et al 2001; Neal 2008).

Studies of cUTI included in this review generally did not present efficacy results stratified according to the specific complicating factor(s). One exception is the trial performed by Peterson et al (2008), which reported significantly different microbiologic eradication rates among the catheterized and non-catheterized patients. These results suggest that the presence of catheters may influence outcomes in these studies, and stratification of results by catheter usage is warranted. We agree with others who have recommended that studies including patients with cUTI should report results stratified according to specific patient characteristics, since patients with cUTI present with a heterogeneous collection of factors that make their infection complicated (Naber 1999).

All of the clinical studies presented compared levofloxacin with another fluoroquinolone. All studies reported similar microbiologic and clinical responses for these comparators. No data are available regarding a comparison of levofloxacin with other classes of antimicrobials. In general, this may be due to a lack of suitable oral alternatives. While direct comparisons are lacking, it has been noted that similar microbiologic success rates ranging from $71 \%-91 \%$ have been reported in trials using beta-lactams and monobactams (piperacillin/tazobactam, ceftriaxone, aztreonam, ceftazidime), carbapenems (imipenem, meropenem, ertapenem) and aminoglycosides (amikacin) (Melekos et al 1991; Nowe 1994; Cox et al 1995; Wells et al 2004). It is not possible to directly compare the results of these studies to the included levofloxacin studies, but crude efficacy rates were similar. Additional research using non-fluoroquinolone comparators would provide not only direct comparisons of efficacy, but would also provide for direct comparisons in safety profiles, which may vary to a greater extent between antimicrobial classes.

Levofloxacin was generally well-tolerated in all of the reviewed trials; this is consistent with what has been observed in other settings at the currently approved doses (Anderson and Perry 2008). A pooled analysis of patients with respiratory tract infections who were prescribed levofloxacin $500 \mathrm{mg}$ or $750 \mathrm{mg}$ found that patients receiving both doses experienced a similar rate of adverse events during treatment, and the specific adverse events observed were similar to those in the trials reviewed here (Khashab et al 2006). Additional adverse events not commonly reported in the reviewed publications, but that have been reported in association with levofloxacin, include photosensitivity, QTc prolongation, hypersensitivity, convulsions, Clostridium difficile-associated diarrhea, and tendon rupture; many of these adverse reactions are rarely reported and have been associated with other fluoroquinolones (Anderson and Perry 2008). A comprehensive review of adverse events associated with fluoroquinolone use is not included here; the reader is referred to a recent review of this topic (Mehlhorn and Brown 2007).

Two of the trials that we reviewed included patients who received altered levofloxacin doses based upon renal function, although results were not stratified according to the dose received (Klimberg et al 1998; Richard et al 1998b). An inherent difference in clinical or microbiologic response would not be expected in these patient groups, assuming that dosing according to renal function was performed appropriately. However, further evidence of the efficacy of the various approved dosing regimens of levofloxacin is needed. Similarly, the trial by Peterson et al (2008) did not report outcomes that were stratified according to whether patients received oral or intravenous therapy. Based on levofloxacin's oral bioavailability of $100 \%$, we do not expect that patients receiving oral levofloxacin would experience poorer outcomes than those receiving intravenous therapy; however, receipt of intravenous therapy may be a marker of more severe illness (eg, bacteremic patients) and thus a more thorough analysis of efficacy that is stratified by the route of administration is warranted.

The majority of the patients included in the trials described in this review presented with infections of moderate severity, and most studies included only patients who received oral therapy on an outpatient basis. The clinical and microbiologic efficacy of levofloxacin in patients with more severe illness (including hospitalized patients) requires 
further study. Furthermore, larger studies are needed so that levofloxacin's efficacy can be evaluated among patients infected with organisms other than $E$. coli, since the incidence of antimicrobial resistance in urinary tract infections caused by these pathogens is not well-described, and cUTI and AP are frequently caused by pathogens other than $E$. coli (Nicolle 1997).

Furthermore, few studies provided meaningful information regarding the development of fluoroquinolone resistance. Although fluoroquinolone resistance in such uropathogens as E. coli remains somewhat rare in the US, the prevalence of such resistance is undoubtedly increasing. Regional variations in the prevalence of levofloxacin resistance have already been identified within the US, where the prevalence of resistance varies from 3.7\% in New England to $9.1 \%$ in the Mid-South region (David et al 2005). Peterson et al (2008) was the only publication to report data on resistance that emerged during the course of therapy, though they reported zero occurrences of this event. Fluoroquinolone-resistant $E$. coli has also been isolated in the rectal flora of patients who received ciprofloxacin for acute uncomplicated cystitis; thus, evaluating resistance in urinary isolates alone may be insufficient (Gupta et al 2005). With the recent proliferation of TMP/SMX-resistant E. coli, fluoroquinolones have frequently become the recommended empiric therapy of choice for acute uncomplicated cystitis (Le and Miller 2001; Warren 2001). In fact, a recent study found that fluoroquinolones had replaced TMP/SMX nationwide as the preferred first-line antimicrobial for uncomplicated cystitis (Kallen et al 2006). Unfortunately, the long-term viability of this strategy in the face of increasing fluoroquinolone resistance is uncertain, and investigations of fluoroquinolone-sparing regimens for cUTI and AP, using agents such as TMP/SMX, are needed (Kallen et al 2006; Gupta et al 2007). The future use of fluoroquinolones in the management of cUTI and AP is also threatened by the ongoing increase in the number of these infections that are caused by ESBL-producing Enterobacteriaceae, which tend to display cross-resistance to fluoroquinolones (Muratani and Matsumoto 2006; Baudry et al 2008). Furthermore, E. coli isolates may harbor resistance-conferring mutations yet appear to be fully-susceptible according to susceptibility breakpoint standards established by the Clinical and Laboratory Standards Institute (Komp Lindgren et al 2003). New trials that examine levofloxacin in the setting of cUTI and/or AP should include data regarding the prevalence of resistance (whether it is present in the original infecting isolate or develops during fluoroquinolone therapy), as it is a mitigating factor in treatment failure, and ongoing surveillance of antimicrobial resistance in uropathogens is of paramount importance.

In the case of uncomplicated urinary tract infections, significant research has led to reduced durations of antimicrobial therapy in patients with these infections. Reducing unnecessarily long durations of therapy is one aim of the judicious antimicrobial movement, which aims to reduce the evolutionary selective pressures that drive the emergence and growing prevalence of antibiotic resistance. In 2007, the US FDA approved a higher dose, shorter duration levofloxacin regimen for cUTI and AP. We identified only one trial, sponsored by Ortho-McNeil Pharmaceuticals, Inc., in which this therapy was evaluated. Although both microbiologic and clinical efficacy results were slightly lower for this regimen ( $750 \mathrm{mg}$ daily for 5 days) than had been reported in other trials using longer durations of lower-dose therapy, this trade-off may be acceptable in patients with less severe disease and may help to limit the increasing development of fluoroquinolone resistance.

\section{Conclusions}

Levofloxacin has demonstrated high rates of microbiologic and clinical success, with outcomes similar to those obtained by comparator fluoroquinolones in the included trials. All of the available fluoroquinolones possess activity against common uropathogens, including E. coli, and most of these agents display favorable pharmacokinetics in the setting of UTI. Alternatives to the fluoroquinolones for the treatment of cUTI and AP include penicillins, cephalosporins, and TMP/SMX; little data regarding the comparative efficacy of these agents is available. It is difficult to assess the generalizability of studies of cUTI and/or AP because of the lack of a standardized definition of cUTI, a high degree of variability in study design, and the level of stratification with which data are reported. An important clinical outcome that was not adequately reported in the majority of these publications was the development of antimicrobial resistance while receiving therapy. This may be an important distinguishing factor among antimicrobials. Ongoing surveillance for fluoroquinolone resistance among uropathogens is needed so that these data can be applied in the empiric therapy decision-making process. Furthermore, more research is needed to determine optimal treatment durations and dosing regimens for antibiotics in the treatment of cUTI and AP. Rational antibiotic use for high-incidence infections, such as the UTIs, are critical to the control of increasing antibiotic resistance (David et al 2005). Although levofloxacin has been associated with favorable outcomes in patients with AP and cUTI, further comparative trials are needed to establish the 
role of levofloxacin in comparison to alternative antimicrobial classes in the management of these infections.

\section{Disclosures}

None of the authors has any conflicts of interest to disclose.

\section{References}

Anderson RU. 1996. Treatment of complicated and uncomplicated urinary tract infections. Antibiotic therapy in urology. S. G. Mulholland, Philadelphia: Lippincott-Raven Publishers. p 23-37.

Anderson VR, Perry CM. 2008. Levofloxacin:a review of its use as a highdose, short-course treatment for bacterial infection. Drugs, 68:535-65.

Baudry PJ, Nichol K, DeCorby M, et al. 2008. Comparison of antimicrobial resistance profiles among extended-spectrum-beta-lactamase-producing and acquired AmpC beta-lactamase-producing Escherichia coli isolates from Canadian intensive care units. Antimicrob Agents Chemother, 52:1846-9.

Bundrick W, Heron SP, Ray P, et al. 2003. Levofloxacin versus ciprofloxacin in the treatment of chronic bacterial prostatitis: a randomized doubleblind multicenter study. Urology, 62:537-41.

Chang TM, Lu PL, Li HH, et al. 2007. Characterization of fluoroquinolone resistance mechanisms and their correlation with the degree of resistance to clinically used fluoroquinolones among Escherichia coli isolates. J Chemother, 19:488-94.

Chenia HY, Pillay B, Pillay D. 2006. Analysis of the mechanisms of fluoroquinolone resistance in urinary tract pathogens. J Antimicrob Chemother, 58:1274-8.

Cox CE, Holloway WJ, Geckler RW. 1995. A multicenter comparative study of meropenem and imipenem/cilastatin in the treatment of complicated urinary tract infections in hospitalized patients. Clin Infect Dis, 21:86-92.

Czaja CA, Scholes D, Hooton TM, et al. 2007. Population-based epidemiologic analysis of acute pyelonephritis. Clin Infect Dis, 45:273-80.

David RD, DeBlieux PM, Press R. 2005. Rational antibiotic treatment of outpatient genitourinary infections in a changing environment. $\mathrm{Am}$ J Med, 118(Suppl 7A): 7S-13S.

Davis R, Bryson HM. 1994. Levofloxacin. A review of its antibacterial activity, pharmacokinetics and therapeutic efficacy. Drugs, 47:677-700.

Drusano GL, Preston SL, Van Guilder M, et al. 2000. A population pharmacokinetic analysis of the penetration of the prostate by levofloxacin. Antimicrob Agents Chemother, 44:2046-51.

Fish DN, Chow AT. 1997. The clinical pharmacokinetics of levofloxacin. Clin Pharmacokinet, 32:101-19.

Foxman B. 2002. Epidemiology of urinary tract infections: incidence, morbidity, and economic costs. Am J Med, 113(Suppl 1A):5S-13S.

Grabe M, Bishop MC, Bjerklund-Johansen TE, et al. 2008. Guidelines on the management of urinary and male genital tract infections, European Association of Urology.

Gupta K, Hooton TM, Roberts PL, et al. 2007. Short-course nitrofurantoin for the treatment of acute uncomplicated cystitis in women. Arch Intern Med, 167:2207-12.

Gupta K, Hooton TM, Stamm WE. 2005. Isolation of fluoroquinoloneresistant rectal Escherichia coli after treatment of acute uncomplicated cystitis. J Antimicrob Chemother, 56:243-6.

Gupta K, Hooton TM, Wobbe CL, et al. 1999a. The prevalence of antimicrobial resistance among uropathogens causing acute uncomplicated cystitis in young women. Int J Antimicrob Agents, 11:305-8.

Gupta K, Scholes D, Stamm WE. 1999b. Increasing prevalence of antimicrobial resistance among uropathogens causing acute uncomplicated cystitis in women. JAMA, 281:736-8.

Hooton TM, Stamm WE. 1991. Management of acute uncomplicated urinary tract infection in adults. Med Clin North Am, 75:339-57.
Kallen AJ, Welch HG, Sirovich BE. 2006. Current antibiotic therapy for isolated urinary tract infections in women. Arch Intern Med, 166:635-9.

Khashab MM, Xiang J, Kahn JB. 2006. Comparison of the adverse event profiles of levofloxacin $500 \mathrm{mg}$ and $750 \mathrm{mg}$ in clinical trials for the treatment of respiratory infections. Curr Med Res Opin, 22:1997-2006.

Klausner, HA, Brown P, Peterson J, et al. 2007. A trial of levofloxacin $750 \mathrm{mg}$ once daily for 5 days versus ciprofloxacin $400 \mathrm{mg}$ and/or 500 $\mathrm{mg}$ twice daily for 10 days in the treatment of acute pyelonephritis. Curr Med Res Opin, 23:2637-45.

Klimberg IW, Cox CE 2nd, Fowler CL, et al. 1998. A controlled trial of levofloxacin and lomefloxacin in the treatment of complicated urinary tract infection. Urology, 51:610-5.

Komp Lindgren P, Karlsson A, Hughes D. 2003. Mutation rate and evolution of fluoroquinolone resistance in Escherichia coli isolates from patients with urinary tract infections. Antimicrob Agents Chemother, 47:3222-32.

Langtry HD, Lamb HM. 1998. Levofloxacin. Its use in infections of the respiratory tract, skin, soft tissues and urinary tract. Drugs, 56:487-515.

Lautenbach E, Strom BL, Bilker WB. 2001. Epidemiological investigation of fluoroquinolone resistance in infections due to extended-spectrum beta-lactamase-producing Escherichia coli and Klebsiella pneumoniae. Clin Infect Dis, 33:1288-94.

Le TP, Miller LG. 2001. Empirical therapy for uncomplicated urinary tract infections in an era of increasing antimicrobial resistance: a decision and cost analysis. Clin Infect Dis, 33:615-21.

Matsumoto T, Takahashi K, Manabe N, et al. 2001. Urinary tract infection in neurogenic bladder. Int J Antimicrob Agents, 17:293-7.

Mazzulli T. 2001. Antimicrobial resistance trends in common urinary pathogens. Can J Urol, 8(Suppl 1):2-5.

McCue JD. 1999. UTIs in at-risk patients: are they 'complicated'? Infect Med, 16:533-40.

Mehlhorn AJ, Brown DA. 2007. Safety concerns with fluoroquinolones. Ann Pharmacother, 41:1859-66.

Melekos MD, Skoutelis A, Chryssanthopoulos C, et al. 1991. A comparative study on aztreonam, ceftazidime and amikacin in the treatment of complicated urinary tract infections. J Chemother, 3:376-82.

Morrissey I, Hoshino K, Sato K, et al. 1996. Mechanism of differential activities of ofloxacin enantiomers. Antimicrob Agents Chemother, 40:1775-84.

Muratani T, Matsumoto T. 2006. Urinary tract infection caused by fluoroquinolone- and cephem-resistant Enterobacteriaceae. Int $J$ Antimicrob Agents, 28(Suppl 1):S10-3.

Naber KG. 1999. Experience with the new guidelines on evaluation of new anti-infective drugs for the treatment of urinary tract infections. Int $J$ Antimicrob Agents, 11:189-96; discussion 213-6.

Naber KG. 2001. Which fluoroquinolones are suitable for the treatment of urinary tract infections? Int J Antimicrob Agents, 17:331-41.

Naber KG, Roscher K, Botto H, et al. 2008. Oral levofloxacin $500 \mathrm{mg}$ once daily in the treatment of chronic bacterial prostatitis. Int J Antimicrob Agents, 32:145-53.

Neal DE Jr. 2008. Complicated urinary tract infections. Urol Clin North Am, 35:13-22.

Nicolle LE. 1997. A practical guide to the management of complicated urinary tract infection. Drugs, 53:583-92.

Nowe P. 1994. Piperacillin/tazobactam in complicated urinary tract infections. Intensive Care Med, 20(Suppl 3): S39-42.

Peng MY. 1999. Randomized, double-blind, comparative study of levofloxacin and ofloxacin in the treatment of complicated urinary tract infections. J Microbiol Immunol Infect, 32:33-9.

Peterson J, Kaul S, Khashab M, et al. 2008. A double-blind, randomized comparison of levofloxacin $750 \mathrm{mg}$ once-daily for five days with ciprofloxacin 400/500 mg twice-daily for 10 days for the treatment of complicated urinary tract infections and acute pyelonephritis. Urology, 71:17-22.

Richard GA, Childs SJ, Fowler CL, et al. 1998a. Safety and efficacy of levofloxacin versus ciprofloxacin in complicated urinary tract infections in adults. Pharm Ther, 23:534-40. 
Richard GA, Klimberg IN, Fowler CL, et al. 1998b. Levofloxacin versus ciprofloxacin versus lomefloxacin in acute pyelonephritis. Urology, 52:51-5.

Richard GA, Mathew CP, Kirstein JM, et al. 2002. Single-dose fluoroquinolone therapy of acute uncomplicated urinary tract infection in women: results from a randomized, double-blind, multicenter trial comparing single-dose to 3-day fluoroquinolone regimens. Urology, 59:334-9.

Ronald AR, Harding GK. 1997. Complicated urinary tract infections. Infect Dis Clin North Am, 11:583-92.

Rubin RH, Shapiro ED, Andriole VT, et al. 1992. Evaluation of new antiinfective drugs for the treatment of urinary tract infection. Infectious Diseases Society of America and the Food and Drug Administration. Clin Infect Dis, 15(Suppl 1):S216-27.

Sobel JD, Kaye D. 2005. Urinary tract infections. In: Mandell GL, Douglas RG, Bennett JE, et al. (eds). Mandell, Douglas, and Bennett's principles and practice of infectious diseases. New York: Elsevier/Churchill Livingstone. 1:875-905.

US Department of Health and Human Services, Food and Drug Administration and Center for Drug Evaluation and Research (CDER). 1998. Guidance for Industry. Complicated urinary tract infections and pyelonephritis-developing antimicrobial drugs for treatment. Clin-Anti. Rockville, MD, Drug Information Branch. Division of Communications Management.
Warren JW. 2001. Practice guidelines for the treatment of uncomplicated cystitis. Curr Urol Rep, 2:326-9.

Warren JW, Abrutyn E, Hebel JR, et al. 1999. Guidelines for antimicrobial treatment of uncomplicated acute bacterial cystitis and acute pyelonephritis in women. Infectious Diseases Society of America (IDSA). Clin Infect Dis, 29:745-58.

Wells WG, Woods GL, Jiang Q, et al. 2004. Treatment of complicated urinary tract infection in adults: combined analysis of two randomized, double-blind, multicentre trials comparing ertapenem and ceftriaxone followed by appropriate oral therapy. J Antimicrob Chemother, 53(Suppl 2):ii67-74.

Xiong Z, Zhu D, Wang F, et al. 2002. Investigation of extended-spectrum beta-lactamase in Klebsiellae pneumoniae and Escherichia coli from China. Diagn Microbiol Infect Dis, 44:195-200.

Zhanel GG, Hisanaga TL, Laing NM, et al. 2006. Antibiotic resistance in Escherichia coli outpatient urinary isolates: final results from the North American Urinary Tract Infection Collaborative Alliance (NAUTICA). Int J Antimicrob Agents, 27:468-75. 
